\title{
Reverse Queries in DATR*
}

\author{
Hagen Langer \\ University of ()snabrïck, Germany \\ hlanger((i)jupiter.r\%.uni-osnabrueck.de
}

\begin{abstract}
DAT'R is a declarative representation language for lexical information and as such, in principle, nentral with respect to particular processing strategies. Previons DATR compiler/interpreter systems support only one access strategy that closely resembles the set of inference rules of the procedural somantics of J) A'T'R (Lvans \& Gazdar 1989a). In this paper wo present an alternattive access strategy (reverse query strutegy) for a nontrivial subset, of DA'T'R.
\end{abstract}

\section{The Reverse Query Problem}

DA'TR (Fvans \& Gazdar 1989a) hals become one of the most widely used formal languages for the representation of lexical information. DA'LR applications have been developed for a wirle variety of languages (including Finglish, Japanese, Kikuyu, Arabic, Tatin, and othors) and many different subclomains of lexical representation, including inflectional morphology, underspecification phonology, non-concatconative morphophonology, lexical semantics, and tone systems ${ }^{1}$.

We presuppose that the reader of the present paper is familiar with the basic features of J)A'T as specified in Evans \& Gazdar [1989i].

The adequacy of a lexicon representation formalism deponds basically on two major factors:

- its declaratine expressiveness: is the fomalism, in principle, capable of representing the phenomena in

"This research was partly supported by the German f'ederal Ministry of Research and 'Tedhology (BMA'l', project VhiRISMOBIL) at the University of Biclefeld. I would like to thank l)afydel Gibbon for very tseful comments on an earlier draft of this payer.

${ }^{1}$ See Cahill [1993], Gibbon [1992], Crazdas [1992], and Kilbury [1992] for recont IDATR applications in these areas. $A_{n}$ informal introduction to DSTlR is given in Gakdar [1990]. 'T'he stanclard

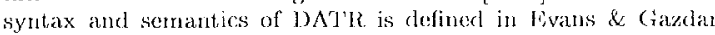
[1989a, 1989h]. Implementation issues are discussed in (ibhon \& Aloua [1991], Jenkins [1990], and in cribbon [1993]. Moser [1992a, $1902 \mathrm{~h}, 1902 \mathrm{c}, 1992 \mathrm{~d}]$ provides interesting insights into the formal properties of 1 ) AT'R (see also the l) A'L'R representations of finite state attomata, different kinds of logics, register operations etc. in Jvans \& (iazdar [1990], and langer [1993]). Andry ot al. [1993] describe how J $\Lambda^{\prime} \mathrm{T} R$ can be used in spech-oriented applications. question, and doess it allow for an explicit troatment of generalisations, subgeneralisations, and ex.ceptionss?

- its range of accessing strategies: are there accessing strategies for all applications which presuppose a lexicon (e.g. parsing, gencration, ...), and do they sup)port the development, maintenance, and evoluation of lexica in an adecyate mannor?

Most of the previous work on DATR has focussed on the former set of criteria, i.e. the declarative features of the language, its expressive capabilitios, and its artequacy for the re-formulation of pre-theoretic informal linguistic concepts. This paper is mainly concorned with the latier set of criteria of adequacy. However, in the case of ISATR, the limited aceess in only one direction has led to a somewhat procedural view of the langlage which, in particular cases, has also had an impact on the cleclarative representations themselves. 1) $\Lambda$ L'R has often been characterised as a functional and deterministic language. 'These features are, of course, not properties of the language itsolf, but rather of the language together wilh a particular procedural interpretation. Actually, the tem deterministic is not applicable to a declarative language, but only makes sense if applica to a procedural language or a particular procodural interpretalion of a language. The DA'lR in. terpreter/compiler systems developed so far ${ }^{2}$ have in common that they support only ono way of accessing the information represented in a DA'L'R thoory. 'This access strabegy, which we will refer to as the standard procedural incerpretation of DATR, closely resembless the inferenee rules clefined in Evans \& Gazdar [1989a]. liven if one considers DA'TR neither as a tool for parsing nor for gencration tasks, but rather as a purely representational device, the one-way-only acecess to D $\Lambda^{\prime} \Gamma^{\prime} R$ theories turns out to be one of the major drawbakes of the model.

One of the claims stated for DATR in Fvans \& Ga\%dar [1989] is that it is computationally tractable. But for many practical purposes, including lexicon development and evaluation, it, is not sufficient that there is any

${ }^{2}$ DA'TlR implementations have been developed by R. Fivans

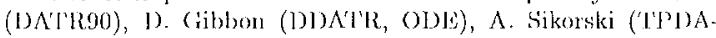
TRRS), J. Kilbury (QI)ATR), G. Drexel (YADL), M. Duda (IIUB $10 \Lambda^{\prime}[R)$, and others. 
arbitrary accessing strategy at all, but there should be an appropriate way for accessing whatever information that is necessary for the purpose in question. This is a strong motivation for investigating alternative strategies for processing IAI'R representations. This paper is concerned with the reverse query problem, i.c. the problem how a given DATR value can be mapped onto the queries that evaluate to it. A standard query consists of a node and a path, e.g. Sheep:<orth plur>, and evaluates to a sequence of atoms (value), e.g. sheep. A reverse query, on the other hand, starts with the value, e.g. sheep, and queries the set of node-path pairs which evaluate to it, for instance, Sheep:<orth sing $>$ and Sheep:<orth plur>. Our solution can be be regarded as an inversion of the parsing-as-deduction approach of the logic programming tradition, since we treat reversequery theorem proving as a parsing problem. We adopt a wellknown strategy from parsing technology: we isolate the context-free "backbone" of DATR and use a modified chart-parsing algorithm for CF-PSG as a theorem prover for reverse queries.

For the purposes of the present paper we will introduce a DATR notation that slightly differs from the standard notation given in Evans \& Gazdar [1989] in the following respects:

- the usual DATR abbreviation conventions are spelled out

- the global environment of a DATR descriptor is cxplicitly represented (even if it is uninstantiated)

- each node-path pair $N: P$ is associated with the set of extensional suffixes of $N: P$ that are defined within the DATR theory

In standard DATR notation, what one might call a non-terminal symbol, is a node-path pair (or an abbreviation for a node-path pair). In our notation a $D A T R$ nonterminal symbol is an ordered set $\left[N, P, C, N^{\prime}, P^{\prime}\right]$. $N$ and $N^{\prime}$ are nodes or variables ranging over nodes. $P$ and $P^{\prime}$ are paths or variables ranging over paths. $C$ is the set of path suffixes of N:P.

A DATR terminal symbol of a theory $\theta$ is an atom that has at least one occurence in a sentence in $\theta$ where it is not an attribute, i.e. where it does not occur in a path.

The suffix-set w.r.t. a prefix $p$ and a set of sequences $S$ (written as $\sigma(p, S)$ ) is the set of the remaining suffixes of strings in $S$ which contain the prefix $p: \sigma(p, S)=$ $\left\{s \mid p^{\wedge} s \in S\right\}$.

Let $N: P$ be the left hand side of a DATR sentence of some DATR theory $\theta$. Let be $\Pi$ the set of paths occurring under node $N$ in $\theta$. The path extension constraint of $P$ w.r.t. $N$ and $\theta$ (written as $C(P, N, \theta)$, or simply $C)$ is defined as: $C(P, N, \theta)=\sigma(P, \Pi)$.

Thus, the constraint of a path $P$ is the set of path suffixes extending $P$ of those paths that have $P$ as a prefix. Example: Consider the DATR theory $\theta$ :
N :

$$
\begin{aligned}
& \langle\rangle==0 \\
& \langle a\rangle==1 \\
& \langle a \text { b }\rangle==2 .
\end{aligned}
$$

The constraint of \langle\rangle (w.r.t. $N$ and $\theta$ ) is $\{\langle\mathrm{a}\rangle,\langle\mathrm{a}$ $b\rangle\}$, the constraint of $\langle a\rangle$ is $\{\langle b\rangle\}$, and the constraint of $\langle a b\rangle$ is $\emptyset$.

We say that a sequence $S=s_{1} \ldots s_{n}(1 \leq \mathrm{n})$ satisfies a constraint $C$ iff $\left\{x \in C \mid x^{\wedge} X=S\right\}=\emptyset$ (i.e. a sequence $S$ satisfies a constraint $C$ iff there is no prefix of $S$ in $C)$.

Now having defined some basic notions, we can give the rules that map standard DATR notation onto our representation:

\section{Mapping rules}

$$
\begin{aligned}
& \mathrm{N}: \mathrm{P}==0 \quad \Rightarrow \quad\left[\mathrm{N}, \mathrm{P}^{\mathrm{P}}, \mathrm{C}, \mathrm{N}^{\prime}, \mathrm{P}^{\prime}\right] \rightarrow \varepsilon \\
& \mathrm{N}: \mathrm{P}==\text { atom } \Rightarrow\left[\mathrm{N}, \mathrm{P}, \mathrm{C}, \mathrm{N}^{\prime}, \mathrm{P}^{\prime}\right] \rightarrow \text { atom } \\
& \mathrm{N}: \mathrm{P}^{\prime}==\mathrm{N}_{2}: \mathrm{P}_{2} \mathrm{P}_{2} \Rightarrow\left[\mathrm{N}, \mathrm{P}, \mathrm{C}, \mathrm{N}^{\prime}, \mathrm{P}^{\prime}\right] \rightarrow\left[\mathrm{N}_{2}, \mathrm{P}_{2}, \mathrm{C}^{\prime}, \mathrm{N}^{\prime}, \mathrm{P}^{\prime}\right] \\
& \mathrm{N}: P=\mathrm{N}_{2} \quad \Rightarrow \quad\left[\mathrm{N}, \mathrm{P}^{\prime}, \mathrm{C}^{\prime}, \mathrm{N}^{\prime}, \mathrm{P}^{\prime}\right] \rightarrow\left[\mathrm{N}_{2}, \mathrm{P}, \mathrm{C}, \mathrm{N}^{\prime}, \mathrm{P}^{\prime}\right]
\end{aligned}
$$

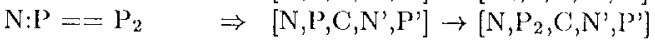

$$
\begin{aligned}
& \mathrm{N}: \mathrm{P}=={ }^{2} \mathrm{~N}_{2}: \mathrm{P}_{2}{ }_{2} \Rightarrow\left[\mathrm{N}, \mathrm{P}^{\prime}, \mathrm{C}, \mathrm{N}^{\prime}, \mathrm{P}^{\prime}\right] \rightarrow\left[\mathrm{N}_{2}, \mathrm{P}_{2}, \mathrm{C}, \mathrm{N}_{2}, \mathrm{P}_{2}{ }_{2}\right] \\
& \mathrm{N}: \mathrm{P}==" \mathrm{~N}_{2}{ }^{\prime} \Rightarrow\left[\mathrm{N}, \mathrm{P}, \mathrm{C}, \mathrm{N}^{\prime}, \mathrm{P}^{\prime}\right] \rightarrow\left[\mathrm{N}_{2}, \mathrm{P}^{\prime}, \mathrm{C}, \mathrm{N}_{2}, \mathrm{P}^{\prime}\right] \\
& \mathrm{N}: \mathrm{P}^{\mathrm{P}}=={ }^{\prime} \mathrm{P}_{2} " \quad \Rightarrow\left[\mathrm{N}, \mathrm{P}, \mathrm{C}, \mathrm{N}^{\prime}, \mathrm{P}^{\prime}\right] \rightarrow\left[\mathrm{N}^{\prime}, \mathrm{P}_{2}, \mathrm{C}_{2}, \mathrm{~N}^{\prime}, \mathrm{P}_{2}{ }_{2}\right]
\end{aligned}
$$

Ilow these mapping principles work can perhaps best be clarified by a larger example. Consider the small DATR theory, below, which we will use as an example case throughout. this paper:

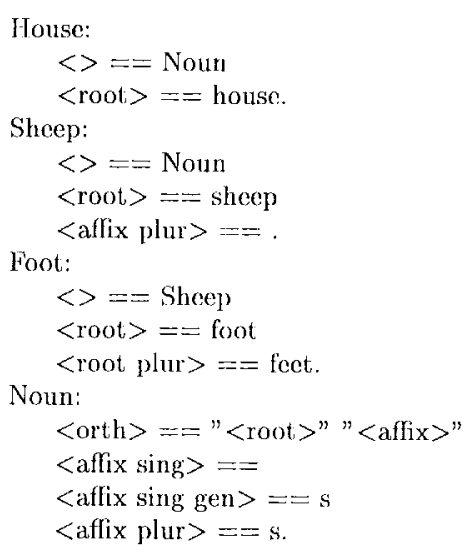

The application of the mapping rules to the DATR theory above yields the following result (unstantiated variables are indicated by bold letters):

$\left[\right.$ House $,<>,\{<$ root $\left.>\}, N^{\prime}, \mathbf{P}^{\prime}\right] \rightarrow\left[\right.$ Noun $,<>,\{<$ root $\left.>\}, N^{\prime}, \mathbf{P}^{\prime}\right]$ [House, $<$ root $\left.>,\{\}, N^{\prime}, \mathbf{P}^{\prime}\right] \rightarrow$ house

$\left[\right.$ Sheep $,<>,\{<$ root $>,<$ affix plur $\left.>\}, \mathbf{N}^{\prime}, \mathbf{P}^{\prime}\right] \rightarrow$ $\left[\right.$ Noun, $<>,\{<$ root $>,<$ affix plur $\left.>\}, \mathbf{N}^{\prime}, \mathbf{P}^{\prime}\right]$

$\left[\right.$ Shoep,$<$ root $\left.>, \emptyset, \mathbf{N}^{\prime}, \mathbf{P}^{\prime}\right] \rightarrow$ sheep

[Sheep, $<$ aflix plur $\left.>, \emptyset, N^{\prime}, \mathbf{P}^{\prime}\right] \rightarrow \varepsilon$

$\left[\right.$ Foot $,<>,\{<$ rool $>,<$ root plur $\left.>\}, N^{\prime}, \mathbf{P}^{\prime}\right] \rightarrow$ $\left[\right.$ Sheep $,<>,\{<$ root $>,<$ root plur $\left.>\}, N^{\prime}, \mathbf{P}^{\prime}\right]$

$\left[\right.$ ['oot,$<$ root $>,\{<$ plur $\left.>\}, \mathbf{N}^{\prime}, \mathbf{P}^{\prime}\right] \rightarrow$ foot 
$\left[l^{\prime}\right.$ oot, $<$ root plur $\left.>, \emptyset, \mathbf{N}^{\prime}, \mathrm{P}^{\prime}\right] \rightarrow$ feet

$\left[\right.$ Nom,$<$ orth $\left.>, \emptyset, \mathbf{N}^{\prime}, \mathbf{P}^{\prime}\right] \rightarrow\left[\mathbf{N}^{\prime},<\operatorname{root}>, \emptyset, \mathbf{N}^{\prime},<\right.$ root $\left.>\right]$

$\left[\mathbf{N}^{\prime},<\right.$ alfix $>, \emptyset, \mathbf{N}^{\prime},<$ affix $\left.>\right]$

$\left[\right.$ Nonn, $<$ allix sing $>,\{<$ gen $\left.>\}, N^{\prime}, P^{\prime}\right] \rightarrow \varepsilon$

$\left[\right.$ Nom, $<$ allix sing gen $>,\left(\eta, N^{\prime}, \mathbf{P}^{\prime}\right] \rightarrow$ s

$\left[\right.$ Noun, $<$ affix plur $>,\left(\phi, N^{\prime}, \mathbb{P}^{\prime}{ }^{\prime}\right] \rightarrow$ s

The general aim of this (somewhat redunclant) notation is to put everything that is necded for drawing inferences from a sentence (especially its global envirommont. and possibly competing clauses at the same node) into the representation of the sentence itsolf. Similar intesnal representations are used in several DATR implementations.

\section{Inference in DATR}

Both standard inference and reverse query inference can be regarded as complex substitution operations defined for serpences of $D A^{\top} \Gamma R$ teminal and non-terminal symbols which apply if particular matching criteria are satisfiecl. In case of DATR standard procedural semantics, a step of inference is the substitution of a DATR nonterminal by a sequence of DATR terminal and nonterminal symbols. The matring criterion applies to a given DATR query and the left hand sides of the sentences of the DATR theory. If the IHSS of a DATR sentences satisfies the matching criterion, a modified version of the right hand side is substilnted for the JIFS. Since the matching criterion is such that there is at most one sentence in a DATR theory with a matching LHS, DATR standard inference is cleterministic and functional. The starting point of DA'TR standard inference is single nonterminal and the derivation process terminates if a sequence of terminals is obtained (or if there is no IIIS in the theory that satisfies the matching criterion, in which case the process of inference terminates with a failure).

In terms of DAT'R reverse cuery procedural semantics, a step of inference is the substitution of a subsequence of a given sequence of I)ATR terminal and non-torminal symbols by a DATR non-terminal. The matching criterion applies to the subsequence and the right hand sides of the sentences of the DATR theory. If the matiching criterion is satisfied, a nodified version of the LHS of the IDAI'R sentence is substituted for the matching subsequence. In contrast to J)A'l'R standard inference, the matching criterion is such that there might be several DATR sentences in a given theory which satisfy it. DATR leverse query inference is hence neither functional, nor deterministic. Starting point of a reverse query is a sequence of torminals (a value). A derivation terminates, if the substitutions finally yield a single nonterminal with identical local and global enviromment (or if there are no matching sentences in the theory, in which case the derivation fails).
Wo now dofine the matching criteria for DAT'JR teminal symbols, DATR nonterminal symbols and sequences of DATR symbols. 'These matching criteria relate extensional lemmata (i.e. already derived partial analyses) to DATR definitional sentences (i.e. "rules" that may yield a further reduction) w.r.t. a given DATR theory $\theta$.

A terminal symbol t, matches another terminal symbol $t_{2}$ iff $t_{1}=t_{2}$. We also say that $t_{1}$ matches $t_{2}$ with an arbitrary suffix and an empty constraint in order to provide compatibility with the definitions for nonterminals, below.

1. A nonterminal $\left[N, I_{1}^{\prime}, C_{1}, N^{\prime}, I^{\prime}\right]$ matches another nonterminal $\left[N, \Gamma_{2}, C_{2}, N^{\prime}, I^{\prime}\right]$ with a suffix $H_{j}$ and a constraint $C_{2}$ if (a) $P_{2}=P_{1}^{\wedge} E$, and (b) $F$, satisfies $C_{1}$. 2. A nonterminal $\left[N, P_{1}, C_{1}, N^{\prime}, I^{\prime}\right]$ matches another nonterminal $\left[N, I_{2}, C_{2}, N^{\prime}, P^{\prime}\right]$ with an empty suffix and a construint $\sigma\left(P_{1}, C_{2}\right)$ if (a) $P_{1}=P_{2}^{\wedge \wedge} \%$, and (b) Li satisfies $C_{2}$.

Exarnple: The non-terminal symbol $[N o d e,\langle a b\rangle$, $\left\{\langle\mathrm{c}\right.$ d e $\left.>\}, N_{1}^{\prime}, P_{1}^{\prime}\right]$ matches $[$ Node, $\langle a$ b c c d $>$, $\left.\emptyset, N_{2}^{\prime}, P_{2}^{\prime}\right]$ with suffix $S=\langle c \cdot \mathrm{d}\rangle$ and constraint $\emptyset$.

From the definitions, given above, we can derive the matching criterion for sequences:

1. The empty sequence matches the empty sequence with an empty suffix and constraint $(t)$

2. A non-empty sequence of (terminal and nonterminal) symbols $s_{1}^{\prime} \ldots s_{n}^{\prime}(1 \leq \mathrm{n})$ matches another sequence of (terminal and non-torminal) symbols $s . \ldots s_{n}$ with suffix $E$ and constraint $C$ if

(a) for cach symbol $s_{i}(1 \leq i \leq n)$ : $s_{i}^{\prime}$ matches $s_{i}$ with suffix $E$ and constraint $C_{i}$, and

(b) $C=C_{1} \cup C_{2} \ldots \cup C_{n}$.

To put it roughly, this definition requires that the synbols of the sequences mateh one another with the same? (possibly empty) suffix. The resulting constraint of the sequence is the union of the constraints of the symbols.

Example: The string of nonterminal symbols $\left.\left.[\mathrm{N} 1,<\mathrm{a}\rangle, \mathrm{C}_{1}, \mathrm{~N}^{\prime} 1, \mathrm{P}^{\prime} 1\right] \quad[\mathrm{N} 2,<\mathrm{x}\rangle, \mathrm{C}_{2}, \mathrm{~N}^{\prime} 2, \mathrm{P}^{\prime} 2\right]$ matches $\left[N 1,\langle a b\rangle,\{\langle c\rangle,\langle d\rangle\}, N^{\prime} 1, P^{\prime} 1\right][N 2,\langle x b\rangle$, $\left.\{\langle c\rangle\}, N^{\prime} 2, P^{\prime} 2\right\}$ with suffix $\langle b\rangle$ and constraint $\{\langle c\rangle$, $\langle d\rangle,\langle e\rangle\}$. $^{3}$

\footnotetext{
${ }^{3}$ 'The matching eriteria, defined above, do not cover nonterminals with comaluable paths, i.c. paths that include (an arbitrary number of possibly recursively embedded) nonterminals. The matching criterion for nonterminals has to be extenderl in order to accomit for statements with evaluable paths: Let be eval $(\alpha, c, \theta)$ a function that maps a string of DATR terminal and nonterminal symbols $\alpha=A_{1} \ldots A_{n}$ onto a string of IDATR terminals $\alpha^{\prime}$ such that (a) each terminal symbol $A_{i}(1 \leq i \leq n)$ in $\alpha$ is mapped onto itself in $\alpha^{\prime}$, and (b) each nonterminal $A_{j}=\left[N_{j}, P_{j}, C_{j}, N_{j}^{\prime}, I_{j}^{\prime}\right](1 \leq$ $j \leq n)$ in $\alpha$ is mapped onto the sequence $a_{j}^{!} \ldots a_{j}^{m}$ in $\alpha^{\prime}$ such that $N_{j}: P_{j}^{\wedge} e=a_{j}^{1} \ldots a_{j}^{m}$ in 0 . "Mr refers to (recur-
} 


\section{The Algorithm}

Metaphorically, DATR "an be regarded as a formalism that exhibits a context-free lackbono ${ }^{4}$. In anal. ogy to a context-free phrase structure rule, a DATR sentence has a left hand side that consists of exactly one non-terminal symbol (i.e. a node-path pair) and a right hand side that consists of an arbitrary number of non-terminal and terminal symbols (i.e. DATR atoms). In contrast to context-free phrase structure grammar, DATR nonterminals are not atomic symbols, but highly structured complex objects. Additionally, DATR differs from CF-PSG in that there is not a unique start symbol but a possibly infinite set of them (i.e. the set of node-path pairs that, taken as the starting point of a query, yicld a valuc).

Despite these differences, the basic similarity of DATR sentences and CF-PSG rules suggests that, in principle, any parsing algorithm for CF-PSGs could be a suitable starting point for constructing a reverse query algorithm for DATR. The algorithm adopted here is a bottom-up chart parser.

A chart parser is an abstract machine that performs exactly one action. This action is monotonically adding itcms to an abstract data-structure called chart, which might be thought of as a graph with annotated ares (which are also often reforred to as edges) or a matrix. There are basically two different kinds of items:

- inactive items (which represent completed analyses of substrings of the input string)

- active items (which represent incomplete analyses of substrings of the input string)

If one thinks of a chart in terms of a graph structure consisting of vertices connected by arcs, then an item can be defined as a triple: (START, END, LABEL), where START and END are vertices connected by an arc labeled with LABEL. Active and inactive items differ with respect to the structure of the label. Inactive items are labeled with a category representing the analysis of the substring given by the START and END position. An active item is labeled with a category representing the analysis for a substring starting at START and ending at some yot unknown position $\mathrm{X}(\mathrm{END} \leq \mathrm{X})$ and a list of categories that still have to

sive) DATR path extension (cf. Evans \& Gazdar 1989a). Notice that $e$ has no index and thus has to be the same for all nonterminals $A_{j}$. Let $X_{1}=\left[N, P_{1}, C_{1}, N^{\prime}, P^{\prime}\right]$ be a nonterminal symbol including an evaluable path $P_{1}, X_{1}$ matches $\left[N, P_{2}, C_{2}, N^{\prime}, P^{\prime}\right]$ with a suflix $E$ and a constraint $C_{x}$ if (a) eval $\left(P_{1}, H^{\prime}, \theta\right)=\pi$, and (b) $\left[N, \pi^{\wedge} H^{\prime}, C_{1}, N^{\prime}, P^{\prime}\right]$ matches $\left[N, P_{2}, C_{2}, N^{\prime}, l^{\prime}\right]$ with suffix $E^{\prime}$ and constraint $C_{x}$ (according to the matching criteria, defined above).

${ }^{4}$ The similarity of cortain DATR sentences and contextfree phrase structure rules has first been mentioned in Gibbon [1992]. be proven to be proper analyses of a sequence of connected substrings starting at $\mathrm{END}$ and ending at $\mathrm{X}$. For the purpose of processing DATR rather than CFPSGs, each active item is additionally associated with a path suffix. Thus an active item has the structure:

(START,END, CATO, CAT' ${ }_{1} \ldots \mathrm{CAT}_{n}$, SUFFIX) Consider the following examples: the inactive item

$\left(0,1,\left[\right.\right.$ House, $<$ orth sing $>,\{<$ gen $>\}$, House, $\left.\left.\mathbf{P}^{\prime}\right]\right)$ represents the information that the substring of the input string consisting of the first symbol is the value of the query House: $<$ orth sing > (with any extensional path suffix, but not gen) in the global environment that consists of the node House and some still uninstantiated path $\mathbf{P}$ '. The active item $\left(0,1,\left[\right.\right.$ Nom, $<$ orth $>, \emptyset$, House, $\left.\mathbf{P}^{\prime}\right]$, [House, $<$ affix $>, \emptyset$, House, $\left.P^{\prime}\right], \varepsilon$ )

represents the information that there is a partial analysis for a substring of the input string that starts with the first symbol and ends somewhere to the right. This substring is the value of the query Noun:<orth $>$ within the global environment consisting of the node House and some uninstantiated global path $\mathbf{P}$ ', if there is a substring starting from vertex 1 that turns out to be the value of the query House:<affix $>$ in the same global environment House: P'.

The general aim is to get all inactive items la beled with a start symbol (i.e. a DATR nonterminal with identical local and global environment) for the whole string which a derivable from the given granmar. There are different strategies to achieve this. The one we have adopted here is based on a chart-parsing algorithm proposed in Kay [1980].

Here is a brief description of the procedures:

- parse is the main procedure that scans the input, increments the pointer to the current; chart position, and invokes the other procedures

- reduce searches the DATR theory for appropriate rules in order to achieve further reductions of inactive items

- add-epsilon applies epsilon productions

- complete combines inactivo and active items

- add-item adds items to the chart

We will now give a more detailed description of the procedures in a pscudo-code notation (the input arguments of a procedure are given in parentheses after the procedure name). Since the only chart-modifying opcration is carried out as a side effect of the procedurc add-item, thore are no output values, at all.

The procedure parse takes as input arguments a vertex that indicates the current chart position (in the initial state this position is 0 ) and the suflix of the 
input string starting at this position. As long as the remaining suffix of the input string is non-empty, parse calls the procedures add-epsilon, reduce, and complete, increments the pointer to the current chart, position, and starts again with the new carmont vertex.

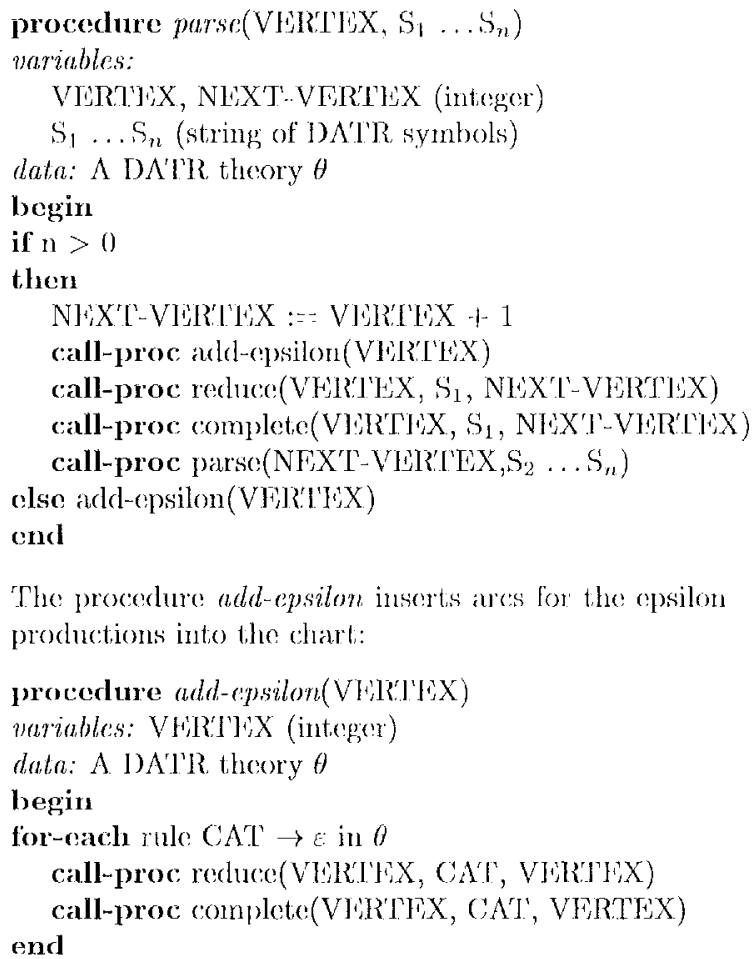

The procedure add-epsilon inserts ares for the epsilon productions into the charl:

The procedure reduce takes an inactive item as the input argurnent and searches the DA'l'R theory for rukes that have a matching left-coruer category. for cach such rule found, reduce itwokes the procedure add-item.

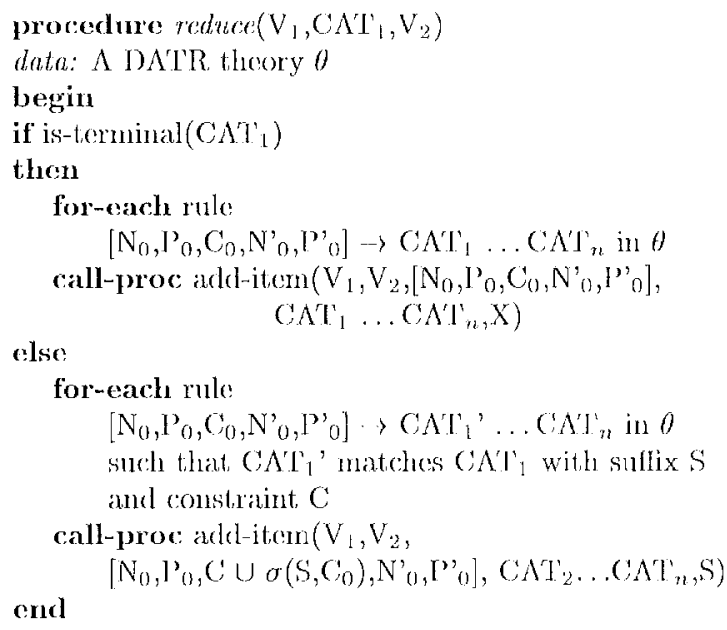

The procedure complete takes an inactive item as an input argument and scarches the chart for active items which can be completed with it. procedure complete $\left(\mathrm{V}_{1}, \mathrm{C} \Lambda \mathrm{T}, \mathrm{V}_{2}\right)$

data: $\Lambda$ shart CII

begin

if is-terminal $\left(C \Lambda^{\prime} \Gamma\right)$

then for-each active itom

$\left(\mathrm{V}_{0}, \mathrm{~V}_{1}, \mathrm{CAl}_{0}, \mathrm{CAT}_{1} \mathrm{CA}^{\prime} \mathrm{I}_{2} \ldots \mathrm{CAT}_{n}, \mathrm{~S}\right)$ in CH

call-proc add-item $\left(\mathrm{V}_{0}, \mathrm{~V}_{2}, \mathrm{M}_{1} \mathrm{CAT}_{2} \ldots \mathrm{CA}^{\prime} \mathrm{T}_{n}, \mathrm{~S}\right)$

else for-each active item

$\left(\mathrm{V}_{0}, \mathrm{~V}_{1},\left[\mathrm{~N}_{0}, \mathrm{P}^{\prime}{ }_{0}, \mathrm{C}_{0}, \mathrm{~N}^{\prime}{ }_{0}, \mathrm{P}^{\prime}{ }_{0}\right], \mathrm{CAT}_{1} \ldots \mathrm{CAT}_{n}, \mathrm{~S}\right)$ in CII such that $C \Lambda^{\prime} \Gamma_{1}$ matches $C, \Lambda \mathrm{T}$ with constraint $\mathrm{C}$ and sullix s

call-proc

add-item $\left(\mathrm{V}_{0}, \mathrm{~V}_{2},\left[\mathrm{~N}_{0}, \mathrm{~L}_{0}, \sigma\left(S, C_{0}\right) \cup \mathrm{C}\right.\right.$,

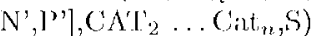

end

The procedure add item is the chart-modifying operation. It takes an active item as an input argument. If this active item has no pending categories, it is regarcled as an inactive iten. In this case add-item inserts a new chart entry for the item, provided it is not already included in the chart, and calls the procedures reduce and complete. If the item is an active item, then it is inserted into the chart, provided it is not alrearly inside.

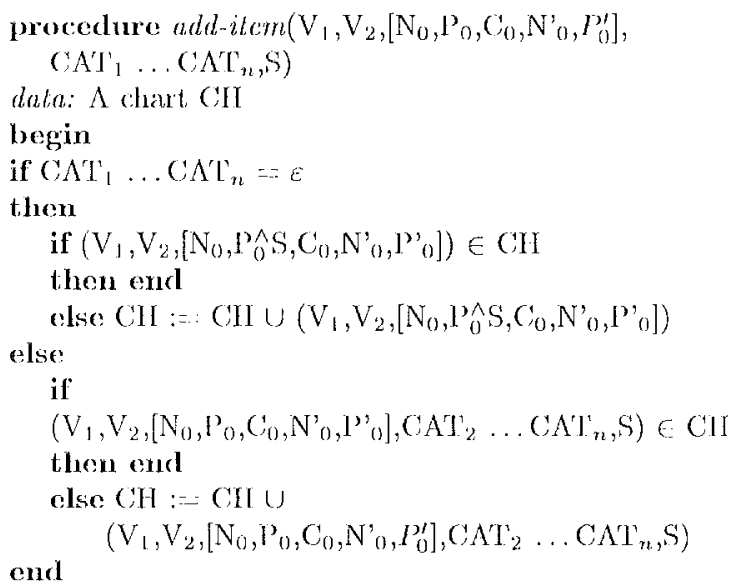

\section{Cycles}

A harel problem for IDAT'R interpreters are cycles, i.c. D ATR statements and sets of I) A'TR statenents which involve recursive definitions such that, standard inference or reverse-query inference does not, necessiarily terminate alter a finite number of steps of inference. Here are some examples of cycles:

- simple c:ycles: $\mathrm{N}:\langle\mathrm{a}\rangle \cdots-<\mathrm{a}\rangle$.

- path lengthening cycles: $\mathrm{N}:\langle a\rangle=-<\mathrm{a} a\rangle$.

- path shortening cycles: $\mathrm{N}:\langle\mathrm{a}$ a $\rangle=-=\langle\mathrm{a}\rangle$. 
While simple cycles have to be considered as semantically ill-formed and thus typically occur as typing errors only, both path lengthening and path shortening cycles occur quite frequently in many DATR represcntations. Note that path lengthening cycles turn out to be path shortening cycles in the reverse query direction, and vice versa. The DATR inforence engine can be prevented from going lost in path-lengthening and path-shortening cycles by a limit on path length. This finite bound on path length can be integrated into our algorithm by modifying the add-item procodure such that only items with a path shorter than the permitted maximum path length are added to the chart.

\section{Complexity}

CF-PSG parsing is known to have a cubic complexity w.r.t. the length of the input string. Though it is crucial for our approach that we exploit the CF-backbone of DATR for computing reverse queries, this result is of no significance, here. DATR is Turing-equivalent (Moser 1992d), and Turing-equivalence has also been shown for a proper subset of DATR (Langer 1993). These theoretical results may a priori outrule DATR as an implementation language for large scale real time applications, but not as a development environment for prototype lexica which can be transformed into efficient task-specific on-line lexica (Andry et al. 1992). With a finite bound on path length our algorithm works, in practice $^{5}$, fast enough to be regarded as a useful tool for the development of small and medium scale lexica in DATR.

\section{Conclusions}

We have proposed an algorithm for the evaluation of reverse queries in DATR. This algorithm makes DATRbased representations applicable for various parsing tasks (e.g. morphological parsing, lexicalist syntactic parsing), and provides an important tool for lexicon development and evaluation in DATR.

\section{References}

[Andry ct al. 1992] François Andry, Norman M. Fraser, Scott McGlashan, Simon Thornton \& Nick J. Youd [1992]: Making DATR Work for Speech: Lexicon Compilation in SUNDIAL. In: Comp. Ling. Vol. 18, No. 3, pages 245-267.

\footnotetext{
${ }^{5} \Lambda$ prolog implementation of the algorithm described in this paper is freely available as a DOS executable program. Please, contact the author for further information.
}

[Cahill 1993] Lynne J. Cahill: Morphonology in the Lexicon. In Sixth Conference of the European Chapter of the Association for Computational Linguistics, pages 87-96, 1993.

[Evans \& Gazdar 1989a] Roger Evans \& Gerald Gazdar: Inforence in DATR. In Fourth Conference of the European Chapter of the Association for Computational Linguistics, pages 66-71, 1989.

[Evans \& Gazdar 1989b] Roger Evans \& Gerald Gazdar: The Somantics of DATR. In: Anthony G Coln [ed.]: Proceedings of the Seventh Conference. of the Society for the Study of Artificial Intelligence and Simulation of Behaviour, pages 79-87,London 1989, Pitman/Morgan Kaufmann.

[Evans \& Gazdar (cds.) 1990] Fvans, Roger \& Gerald Gazdar [eds.]: Tho DATR Papers. Brighton: University of Sussex Cognitive Science Research Papor CSRP 139, 1990.

[Gazdar 1992] Gerald Gazdar: J'aradigm Function Morphology in DATR. In: I,. J. Cahill \& Richard Coates [ods.]: Sussex Papers in General and Computational Linguistics: Presented to the Linguistic Association of Great Britain Conference at Brighton Polytechnic, 6th-8th April 1992. Cognitive Science Research Paper (CSRP) No. 239. University of Sussex, 1992, pages 43-54.

[Gibbon 1992] Iafydd Gibbon: ILEX: A linguistic approach to computational lexica. In: Ursula Klonk [ed.]: Computatio Isinguae. Aufsätze zur algorithmischen und quantitativen Analyse der Sprache, pages $32-53$.

[Gibbon 1993] Dafydd Gibbon: Generalised DATR for flexible access: Prolog specification. English/Linguistics Occasional Papers 8. University of Bielefeld.

[Gibbon \& Ahoua 1991] Dafydd Gibbon \& Firmin Ahoua: DDATR: un logiciel de traitement, d'héritage par défaut pour la modélisation lexical. Chiers Ivoriens de Recherche Linguistique (civl) 27. Université Nationale de Côte d'Ivoire. Abidjan, 1991, pages 5-59.

[Jenkins 1990] Elizabeth $A$. Jenkins: Enhancements to the Sussex Prolog DATR Implementation. In: Evans \& Gazdar [eds.] [1990], pp. 41-61.

[Kay 1980] Martin Kay: Algorithm Schemata and Data Structures in Syntactic Processing. XEROX, Palo Alto.

[Kilbury 1993] James Kilbury: Paradigm-Based Derivational Morphology. In: Günther Görz [cd.]: KONVENS 92. Springer, Berlin etc. 1992, pagess 159-168. 
[Ianger 1993] Hagen Langer: DATR without nodes and global inheritance. In: Proce of 4. Fachtagung Deklarative und prozedurale Aspekte der Sprachverarbeitung der DGfS/CL, University of Iramburg, pages $71-76$.

[Moser 1992a] Iionel Moser: DATR Paths as Arguments. Cognitive Science Research l'aper CSRP 216, University of Sussex, Brighton.

[Moser 1992b] Tiond Moser: Lexical Constraints in DATR. Cognitive Science Research Paper CSRP 215, University of Sussex, Brightom.

[Moser 1992c] Lionel Moser: Evaluation in DATR is co-NP-Hard. Cognitive Science Researde Paper CSRP 240, University of Sussex, Brighton.

[Moser 1992d] Jionol Moser: Simulating 'luring Machines in DATR. Cognitive Science Research Paper CSRP 241, University of Sussex, Brighton. 